\title{
Diagnóstico Afectivo Autopercibido por el Alumnado en las Clases de Educación Física
}

\author{
Affective Diagnosis Self-Perceived by the Students in Physical Education Classes
}

\author{
Pedro Gil-Madrona ${ }^{1}$, Manuel Javier Cijudo-Prado ${ }^{2}$ y Jesús Manuel Martínez-González ${ }^{3}$
}

\begin{abstract}
Resumen
El estudio se ocupa de realizar una evaluación diagnostica de los afectos y de la intensidad de estos que autoperciben los alumnos de Educación Primaria en las clases del área de Educación Física, evaluando los estados afectivos en función del género y de su índice de masa corporal (IMC). La muestra la conforman 786 alumnos de $5^{\circ}$ y $6^{\circ}$ de Educación Primaria. Para evaluar la afectividad se utilizó la versión del PANAS validada en castellano por Sandín (2003). Las puntuaciones son más elevadas en los afectos positivos que en los negativos. Los resultados evidencian diferencias significativas entre chicos y chicas en las variables que más contribuyen al factor de afectos positivos. En cuanto a las diferencias en función del IMC del alumnado en la dimensión afectos positivos, todas las puntuaciones medias más elevadas se encuentran en el alumnado sin problemas de obesidad/sobrepeso. En la dimensión estados afectivos negativos todas las puntuaciones medias más elevadas se encuentran en el alumnado con problemas de obesidad/sobrepeso. Se evidencian diferencias significativas en función del IMC entre alumnos con problemas de sobrepeso/obesidad y alumnos sin problemas de sobrepeso/obesidad en los afectos positivos. No existen diferencias significativas en afectos negativos. Tanto los chicos como las chicas sin problemas de obesidad/sobrepeso presentan más afectos positivos que los chicos y chicas con problemas de obesidad/sobrepeso. En cuanto a los afectos negativos se confirma un patrón diferenciado entre chicos y chicas.
\end{abstract}

Palabras clave: educación física, estados afectivos, obesidad, alumnado, educación primaria

\begin{abstract}
This study is concerned with carrying out a diagnostic evaluation of the effects and intensity that Primary Education students self-perceived in Physical Education lessons, evaluating affective states based on gender and body mass index (BMI). The sample comprises 786 students from the 5th and 6th grades of Primary Education, where, to evaluate affectivity, the version of PANAS validated in Spanish by Sandín (2003) was used. Thus, scores reveal higher for positive than adverse affectlessness. The research results confirm significant differences between boys and girls in the variables that most contribute to the positive affect factor. Regarding the differences based on the BMI of the students in the dimension of positive affects, all the highest mean scores are found in students without obesity/overweight issues. To this extent, negative affection states the highest mean scores in the students with obesity/overweight problems. Hence, there are significant differences according to BMI between students with overweight/obesity problems and students without overweight/obesity problems in positive affects. There are no significant differences though in negative affects. Both boys and girls without obesity/overweight problems show more positive affects than boys and girls with obesity/overweight problems. Regarding negative affects, a differentiated pattern between boys and girls is confirmed.
\end{abstract}

Keywords: physical education, affective states, obesity, students, primary education

\footnotetext{
${ }^{1}$ Profesor Titular de Universidad. Departamento de Didáctica de la Expresión Corporal. Doctor en Ciencias de la Educación. Facultad de Educación de Albacete. Universidad de Castilla La Mancha. España. Albacete. C.P. 02071. Tel.: 0034-967599200 ext. 2530. Correo: pedro.gil@uclm.es

${ }^{2}$ Profesor Contratado Doctor. Departamento de Psicología. Licenciado y Doctor en Psicología. Facultad de Educación de Ciudad Real. Universidad de Castilla La Mancha. España. Correo: manueljavier.cijudo@uclm.es

${ }^{3}$ Facultad de Estadística. Universidad Complutense de Madrid. España. Correo:direccionjesus@ @otmail.com

Revista Iberoamericana de Diagnóstico y Evaluación - e Avaliação Psicológica. RIDEP · N58 · Vol.1 · 5-18 · 2021

ISSN: 1135-3848 print /2183-6051online
} 


\section{Introducción}

Una de las finalidades de la Educación Física escolar es la de promover un estilo de vida activo y sano, y fomentar o crear hábitos de adherencia a la práctica de la actividad física para que los alumnos continúen activos. En esta línea, Van Acker, Carreriro da Costa, Bourdeaudhuij, Cardon y Haerens (2010), bajo el prisma de la teoría social cognitiva, sostienen que los factores ambientales, como el contexto de la clase, la motivación, su contenido y el comportamiento del profesor, influyen directa e indirectamente en los niveles de actividad física durante las clases de Educación Física, así como el interés de los alumnos por esta materia y el disfrute de la misma.

De una parte, los beneficios de la participación en actividades físicas y deportivas están reconocidas por la literatura científica. Numerosos estudios han constatado la influencia que esta asignatura puede llegar a tener en la promoción de estilos de vida saludables (Bailey, 2006; Zach, et al. 2013). De otra, la obesidad constituye uno de los factores principales de riesgo en las enfermedades cardiovasculares; el sobrepeso y la obesidad han sido declarados epidemia global (Malik, Willett, \& Hu, 2013) y en especial en los países desarrollados (Ogden, Carroll, Kit, \& Flegal, 2012), afectando no solo a la población adulta sino también a la infantil y a la adolescente. Estos datos son corroborados por varios trabajos recientes, como el estudio ALADINO (Alimentación, Actividad Física, Desarrollo Infantil y Obesidad, 2011, PerezFarinos, López-Sobaler, Ángeles Dal Re, Villar, Labrado, Robledo, \& Ortega, 2013) y Sánchez, Jiménez, Fernández y Sánchez (2013). La obesidad está relacionada con hábitos alimentarios inadecuados y el sedentarismo (CárdenasCárdenas et al., 2015).

Se han de tomar, por tanto, medidas orientadas a frenar y reducir este problema cada vez más frecuente en la población infantil. Tenemos que actuar ahora, y ser más activos, antes de que se pierda a esta generación en la lucha contra la obesidad y contra las debilitantes enfermedades que de ella se derivan (Perry, 2013) ya que existe una relación positiva entre el hábito de ejercitarse en la niñez y adolescencia y una vida activa en la edad adulta (Hausenblas \& Fallon, 2006; Biro \& Wien, 2010; Morano, Colella, \& Capranica, 2011).

Además de los beneficios físicos que conlleva la actividad física para un crecimiento sano de la infancia, tal actividad produce efectos positivos socio-emocionales y afectivos en la infancia y en la juventud y provoca adherencia a la práctica de la actividad física, lo que conduce a crear estilos de vida activos tanto en la juventud como en la edad adulta (Donnelly, Blair, Jakicic, Manor, Rankin, \& Smith 2009).

Otra gran cantidad de estudios han demostrado que la motivación, entendida como la "intensidad del comportamiento, persistencia, la elección de posibilidades de acción y de rendimiento" (Roberts, 1992, p.6) es un factor crucial que está detrás de la participación en las actividades físicas y deportivas (González-Cutre, Sicilia, \& Moreno, 2008; Ntoumanis, Pensgaard, Martin, \& Pipe, 2004; Yli-Piipari, Watt, Jaakkola, Liukkonen, \& Nurmi, 2009). Asimismo, el disfrute contribuye al aumento de la motivación y, por consiguiente, a la participación en las actividades física. Ello contribuye a que los alumnos intervengan en las actividades físicas, que se impliquen en las tareas y les ayude a la realización de las mismas con éxito. Parece, por tanto, que el disfrute representa un factor clave que subyace a la motivación y por tanto a la participación en Educación Física (Cox, Smith, \& Williams, 2008; Cox \& Ullrich-French, 2010).

De otro lado, actualmente algunos movimientos sociales propugnan que el aprendizaje socioemocional, afectivo y el desarrollo de la inteligencia emocional son vitales para la adaptación social y el bienestar psicológico y físico de las personas (BauselaHerreras, 2018; Bisquerra, Pérez-González, \& García, 2015; Durlak, Weissberg, Dymnicki, Taylor, \& Schellinger, 2011), y la escuela no escapa a estos postulados. Conocerse a sí mismo, ser conscientes de las propias emociones y estados afectivos, de los motivos o causas de nuestros comportamientos, conocer los posibles móviles emocionales en la conducta de los demás, son temas candentes que cada vez interesan más a los profesores, pues sin duda la regulación afectiva solo puede tener lugar en el contexto de una relación con otro ser humano y el niño necesita ir 
aprendiendo a regular sus emociones (Bisquerra et al., 2015; Manríquez-Betanzos \& Montero-López, 2018). Y esas relaciones humanas son una constante durante las clases de Educación Física. PISA en el 2015 incorpora por primera vez la evaluación del bienestar subjetivo en sus evaluaciones y en el 2018 vuelve a evaluar dicha dimensión y anuncia que en la evaluación del 2021 continuará haciéndolo.

$\mathrm{Si}$ nos centramos en estudios previos realizados en relación con la Educación Física, se pueden encontrar diversidad de aportaciones que enfatizan las interrelaciones entre la parte física y mental del ser humano (Ruiz, Lorenzo, \& García, 2013) y la influencia de la expresión corporal en relación con la vivencia emocional (Motos, 2003).

Recientemente se ha relacionado el entusiasmo, la emoción y la cognición con la percepción de la competencia y la actitud hacía la participación en programas de actividad física (Hashim, Grove, \& Whipp, 2008). Parece ser que las experiencias negativas durante la actividad física hacen que las personas se consideren incompetentes en este ámbito (Gutiérrez, 2000).

Espada y Calero (2012) afirman que la asignatura de Educación Física tiene una relación muy directa con los elementos que componen la inteligencia emocional, ya que la empatía, el control de las emociones, la automotivación y las habilidades sociales son elementos que necesariamente van a existir dentro de la práctica deportiva, ya sea competitivo o educativo.

La actividad física puede promover conductas prosociales y las emociones juegan un papel muy importante, tal y como sido mostrado por $\mathrm{y}$ Lavega, March y Filella (2013) y Lavega et al. (2013). Mientras que la literatura muestra que la cognición y el rendimiento han sido factores ampliamente revisados y estudiados, el dominio afectivo todavía no ha originado tantas aportaciones. En este sentido, algunos autores vinculan el área de Educación Física al creciente interés por el trabajo desde un punto de vista emocional y resaltan que es una materia en la cual, dada su idiosincrasia, el alumnado tiene la oportunidad de manifestar intensamente un abanico emocional rico y variado (Durán, Lavega, Salas, Tamarit, \& Invernó, 2015).

Los distintos dominios de acción motriz pueden originar circunstancias asociadas a la activación intensa de emociones positivas. Por contra, determinadas actividades pueden provocar muy pocas emociones negativas con valores intensos (Zamorano-García, Gil-Madrona, PrietoAyuso, \& Zamorano-García, 2018). Esta conclusión es de gran interés para el campo de la Educación Física, ya que una de las razones que explica la inactividad de la población adulta es la valoración negativa que se tiene de las experiencias realizadas en la escuela. De ahí la necesidad del presente estudio. Pieron, García y Ruiz (2007) atestiguan que los estudiantes que participan en actividades físicas y deportivas en edad escolar, así como aquellos que hacen ejercicio físico con regularidad, muestran una actitud mucho más positiva hacia la educación física. Aspectos positivos, tales como la autoestima, la motivación y la actitud emocional correcta promueven el desarrollo y la consolidación de conductas asociadas a una actividad física y a un estilo de vida saludables (Spence \& Blanchard, 2001). Ciertamente como señalan Parlebas (2001) y Lagardera y Lavega (2003) cada juego deportivo constituye un auténtico laboratorio de reacciones emocionales, que hace que cada alumno viva de manera distinta una sesión de Educación Física. La comprensión de la relación existente entre los distintos tipos de juegos deportivos y las distintas clases de emociones es un tema de máxima importancia para el diseño, la aplicación y la evaluación de programas en el campo de la actividad física y el deporte orientados hacia el bienestar personal y social, cuestión esta que ya ha sido estudiada por Lavega et al (2013). En donde observaron que, en cada dominio de acción motriz, se observó el mismo patrón de comportamiento de las emociones. Pero el presente trabajo trata de ahondar o esclarecer los afectos o estados afectivos del alumnado en las clases de Educación Física no según sea el dominio de acción motriz sino desde su subjetividad teniendo en consideración su IMC. No pretende profundizar en qué afectos producen diferentes tipos de juegos motrices en función del dominio de acción motriz o si los participantes actúan motrizmente entre ellos como puede ser en un juego cooperativo, de oposición o de cooperación-oposición.

El interés del presente estudio radica en el hecho de que se ha prestado muy poca atención al 
estudio de las emociones en el ámbito educativo en el entorno de la Educación Física, hasta el momento solo se han llevado a cabo doce estudios (Mujica, Orellana, \& Concha, 2017). Cabría citar, en el perímetro educativo en general, las perspectivas centradas en el entorno socioeconómico de las familias, en el rendimiento y las que se focalizan en factores de naturaleza más objetiva, como la prevención de conductas de riesgo (Brackett \& Mayer, 2003), el bienestar subjetivo (Bar-On, 2006), bienestar personal y laboral (Pena, Extremera, \& Rey, 2011), la robustez de la relaciones familiares (Chan, 2006), el ajuste socioescolar del alumnado (Pena \& Repetto, 2008), la competencia social (Brasseur \& Gregoire, 2011), la salud mental (Bhullar, Shutte, Malouff, 2012) y la felicidad (Bar-On, 2010), entre otros aspectos.

Se hace preciso investigar la labor pedagógica y didáctica de la Educación Física en el alumnado (Gil-Madrona \& Contreras, 2003) y en especial, como es el caso que nos ocupa, en cuanto a las emociones que genera en los alumnos y alumnas, considerando las características del alumno que aprende, así como identificar si el alumnado está motivado para seguir practicando actividad física y reconocer la importancia de sus resultados en el ámbito afectivo (McCaughtry, 2004). Todas las corrientes educativas que pretendan aportar procedimientos adecuados en la enseñanza de la Educación Física, deberían considerar el valor que tiene la vivencia emocional y afectiva Esta vivencia repercute directamente en la experiencia de la actividad física que reciben los jóvenes, así como sobre su futura motivación para participar en programas físicos orientados a mejorar su salud psicológica, física y social. Sin embargo, aún conocemos demasiado poco sobre qué sienten y experimentan ellos en Educación Física. Lo cierto es que si se identifican los factores que pueden ejercer mayor influencia sobre el desarrollo de actitudes tanto positivas como negativas, sería posible prestarles mayor atención a los más susceptibles de generar actitudes positivas y modificar aquellos otros que parecen contribuir al desarrollo de actitudes negativas. Sin duda, las investigaciones realizadas sobre la dimensión afectiva en las clases adquieren una gran importancia para las decisiones que toma el docente en su quehacer pedagógico, ofreciéndole una mayor comprensión del tema.

$\mathrm{Al}$ ser la Educación Física un espacio donde el alumnado se implica en todas sus dimensiones (física-motriz, mental, afectiva y social), dicho espacio se hace favorable para fomentar la educación emocional de la persona. El deporte es un agente de la Educación Física que ofrece muchas posibilidades de acción, el cual, según Bisquerra (2011), este tiene un gran poder para generar emociones.

Por otra parte, desde el punto de vista moral y psicosocial, los estudios de Strong et al. (2005) publicados por la revista The Journal of Pediatrics, afirman que existe una relación positiva entre la práctica de actividad física en el niño y los efectos emocionales positivos, como son el aumento de la autoestima, disminución de la ansiedad y del estrés. Para ello, pretendemos reforzar los atributos positivos del niño $\mathrm{y}$ disminuir los negativos, pero conociendo primero las emociones que experimentan los niños y las niñas teniendo en consideración su IMC (Índice de Masa Corporal).

De ahí que se haya propuesto estudiar las emociones de los alumnos y alumnas y su relación con su IMC en la Educación Física escolar ya que si bien hay algún estudio previo en relación a las emociones en Educación Física (Gil-Madrona \& Martínez-López, 2016) no se ha encontrado ningún estudio en la investigación previa que relacione las emociones, la Educación Física y el índice de masa corporal, proporcionando un enfoque novedoso que centra su atención en los procesos de expresión emocional en la asignatura.

Ello posibilitará, de una parte, explicar la ansiedad de los alumnos y alumnas, la frustración o inseguridad que perciben en determinados momentos y relación o no con su IMC y, por otra, el conocimiento de dicha realidad facilitará al profesorado el desarrollo de medidas orientadas a aumentar el interés de todos los alumnos en las clases de Educación Física. Sin duda, fomentar la motivación de los alumnos, suscitando su interés y sentimientos de competencia ayudará a alcanzar los objetivos de esta materia (Scrabis-Fietcher \& Silverman, 2010). En efecto, dicho conocimiento, posibilitará al profesor de Educación Física la planificación de actividades que conduzcan a la educación emocional, intentando responder a las 
necesidades actuales y que tradicionalmente no se han atendido en las diferentes materias académicas ordinarias (Bisquerra, 2003). Actividades que posibiliten la autoexpresión y el refuerzo de la identidad personal; estrategias para recibir las opiniones, sentimientos, derechos e intereses de forma asertiva; así como actividades que buscan la mera expansión y disfrute del alumno, bloqueando y reduciendo los niveles de estrés, ansiedad y preocupación. Este tipo de estrategias y actividades permitirá trabajar y actuar sobre determinados escenarios emocionales del alumno tales como: no ignorar sus sentimientos y emociones, mantener un entusiasmo constante durante la instrucción, adecuar la interacción a la tarea, estados de infelicidad y tristeza; situaciones de desinterés, desánimo, aburrimiento $\mathrm{y}$, en general, falta de estímulos; situaciones de angustia, miedo, estrés; así como inseguridad o confusión (Gonzalez \& Lleixa, 2010; Pellicer, 2011).

Antes de poner en marcha programas que desarrollen competencias socioemocionales en nuestros alumnos y alumnas en Educación Física, se debe de indagar sobre sus emociones durante el desarrollo de la materia, abordando cuestiones como: ¿Cuáles son los estados emocionales que tienen y experimentan los alumnos y alumnas durante el desarrollo de las clases de Educación Física y la relación existente con su índice de masa corporal en una materia eminentemente procedimental como es la Educación Física?. Pues Marchesi y Hernández (2003) señalan que la abundancia de fracasos en el aprendizaje en diversas edades y niveles educativos puede ser explicada, en gran parte, por la aparición de actitudes negativas, cuya detección será el primer paso para contrarrestar su influencia negativa con efectividad.

Una vez conocidos los principales estudios relacionados con nuestro ámbito de preocupaciones y reflexiones, pasamos a concretar y acotar el objeto de este trabajo. Se pretende indagar, identificar y analizar la intensidad y el tipo de afectos -positivos o placenteros o negativos y displacenteros- experimentadas por los alumnos y alumnas durante la clase de Educación Física a lo largo del desarrollo e impartición de dicha asignatura, así como estudiar la intensidad y el tipo de dichos afectos predominantes en alumnos y alumnas comparando los estados afectivos de ambos grupos, analizando las relaciones, las similitudes o diferencias que pudieran existir entre unos y otras en función de su IMC, en lo que respecta a la intensidad de las emociones y afectos experimentados durante el desarrollo de la asignatura de Educación Física. Se trata de conocer los afectos en niños y niñas que se producen en esta área eminentemente procedimental y su relación con el IMC.

\section{Método}

\section{Participantes}

Para este estudio se empleó un muestreo no probabilístico del tipo incidental o por accesibilidad. La muestra total se compone de 786 alumnos de $5^{\circ}$ y $6^{\circ}$ de educación primaria de cinco localidades. En la distribución por sexos, 406 $(51.65 \%)$ son chicos y $380(48.35 \%)$ son chicas. Por edades, el $18.2 \%$ tiene 10 años; el $45.1 \% 11$ años; los de 12 son el $33.3 \%$ y solo el $3,2 \%$ alcanza los 13 .

\section{Instrumentos de evaluación}

Para evaluar la afectividad se utilizó la versión del PANAS (Positive and Negative Affect Schedule; Watson, Clark, \& Tellegen, 1988) validada en castellano por Sandín (2003) para su uso con niños y adolescentes (PANASN). Se compone de 20 ítems y presenta una estructura bidimensional: afecto positivo (AP) (e.g., "me intereso por la gente o las cosas") y afecto negativo (AN) (e.g.,"siento miedo"), con 10 ítems para cada subescala. El cuestionario es cumplimentado por el niño/adolescente teniendo en cuenta la manera en que éste se siente o comporta habitualmente. En el presente estudio, se incluyó la raíz "indica cómo te sueles sentir al concluir la clase de Educación Física...".

La escala consta de tres alternativas de respuesta, descritas éstas como «Nunca» (1), «A veces» (2), y «Muchas veces» (3). Los datos sobre la fiabilidad y la validez indican que la prueba posee aceptables propiedades, tanto en lo que concierne a la fiabilidad (consistencia interna) como a la validez convergente y divergente (Sandín, 2003). También se tomaron las medidas sobre estatura y peso corporal, para calcular el índice de Masa Corporal (IMC). 


\section{Diseño y procedimiento}

Primero se contactó con los directores de los centros educativos, explicando el proyecto y solicitando la colaboración. Con aquellos que aceptaron colaborar se realizó una entrevista para exponer el proyecto y entregar los consentimientos informados para los padres del alumnado participante. Después de la recepción de los consentimientos de los padres se llevó a cabo la aplicación del instrumento de evaluación nada más concluir la clase de Educación Física, así como las medidas de talla y peso del alumnado. Se garantizó al alumnado el carácter anónimo y confidencial de los datos obtenidos en el presente estudio. Al alumnado se le indicó claramente que se refiere a los afectos y las emociones sentidas en la clase de Educación Física.

La evaluación antropométrica fue realizada por dos investigadores expertos con formación ISAK nivel I. La medición se hizo con los participantes descalzos. Se tomaron medidas de la talla con tallímetro portátil de escala (rango de 0,1 $\mathrm{cm}$ ) modelo Seca 222 (Seca, Alemania), y el peso mediante báscula Tanita BF 522 (Tania Corporation of America Inc.; Illinois, USA). Con ambas informaciones calculamos el IMC, para la edad objeto de estudio, usando la fórmula peso (kgr)/altura (m2).

\section{Plan de análisis de datos}

En primer lugar, se procedió a analizar la fiabilidad (consistencia interna) de cada una de las dimensiones, $A P$ y $A N$, a través del cálculo del alfa de Cronbach $(\alpha)$ y del coeficiente Omega de McDonald $(\Omega)$. Además, se realizó un estudio descriptivo de las respuestas dadas por el alumnado. Posteriormente, se analizaron las correlaciones, mediante el coeficiente de correlación de Pearson entre las puntuaciones correspondientes a las dimensiones $A P$ y $A N$. Además se estudiaron las diferencias mediante análisis de varianza (ANOVA) en función del sexo del alumnado en relación al $A P$ y $A N$. Asimismo, se estudiaron las posibles diferencias existentes en función del IMC del alumnado mediante ANOVA. En este sentido, se utilizaron los criterios de la OMS (2010). Por tanto, el primer grupo se conformó con aquellos alumnos que tienen un peso normal y se denominó grupo de alumnos sin problemas de obesidad/sobrepeso mientras que el segundo quedó compuesto por alumnos con exceso de peso, sobrepeso y obesidad y se denominó grupo con problemas de obesidad/sobrepeso. Finalmente se analizaron las diferencias existentes entre ambos grupos mediante ANOVA en función del IMC del alumnado tanto en chicos como en chicas. En todos los casos, el tamaño del efecto de estas diferencias se calculó mediante el estadístico $d$ de Cohen.

\section{Resultados}

En primer lugar, se presentan las medias, desviaciones típicas, los coeficientes alfa de Cronbach, coeficientes de omega de McDonald y correlaciones para las dimensiones $A P$ y $A N$ utilizadas en este estudio (Tabla 1). En segundo lugar, el análisis de las puntuaciones medias muestra que para los ítems se obtuvieron puntuaciones que se sitúan entre los siguientes valores (2.63-1.38). Como se observa, la puntuación media es más elevada en la dimensión $A P$ que en la dimensión $A N$.

Tal y como se refleja en la Tabla 1, los coeficientes de fiabilidad (consistencia interna) de Cronbach son aceptables, por encima de .70. Del mismo modo, los coeficientes de fiabildiad de McDonald son adecuados. Por otro lado el coeficiente de correlación entre $A P$ y $A N$ fue pequeño y en la dirección esperada ( $r=-.08$; $p=.05$ ) lo que aporta una evidencia empírica más a la estructura bifactorial del instrumento y de la independencia de las dos dimensiones.

Para explorar las posibles diferencias de medias en IMC en función del sexo del alumnado, se crearon dos subgrupos para su comparación

Tabla 1. Matriz de correlaciones y estadísticos descriptivos de las variables de afecto positivo y

\begin{tabular}{|c|c|c|c|c|c|c|}
\hline \multicolumn{7}{|c|}{ negativo } \\
\hline & Media & DT & $\alpha$ & $\Omega$ & 1 & 2 \\
\hline $\begin{array}{l}\text { Afecto } \\
\text { Positivo }\end{array}$ & 24.43 & 3.15 & .70 & .78 & . & \\
\hline $\begin{array}{l}\text { Afecto } \\
\text { negativo }\end{array}$ & 15.96 & 3.79 & .79 & .81 & $-.080^{*}$ & - \\
\hline
\end{tabular}


Tabla 2. Media, desviación típica (DT), ANOVA, y tamaño del efecto de las diferencias de medias (d) en función del sexo del alumnado

\begin{tabular}{lccccccc}
\hline \multicolumn{2}{c}{ Chicos } & \multicolumn{7}{c}{ Chicas } & & & \\
\hline Variables & Media & $D T$ & Media & $D T$ & $F$ & sig & $d$ \\
\hline Afecto Positivo & 24.54 & 3.11 & 24.32 & 3.18 & -.765 & .301 & .07 \\
Interés & 2.42 & .55 & 2.45 & .56 & -.656 & .456 & .05 \\
Animación & 2.43 & .63 & 2.48 & .61 & -.879 & .234 & .08 \\
Energía & 2.68 & .55 & 2.58 & .60 & -1.923 & .002 & .18 \\
Entusiasmo & 2.29 & .62 & 2.37 & .65 & -1.009 & .072 & .13 \\
Orgullo & 2.53 & .57 & 2.53 & .60 & -.010 & .998 & .00 \\
Disposición & 2.39 & .66 & 2.35 & .65 & -.089 & .354 & .06 \\
Inspiración & 2.27 & .65 & 2.21 & .66 & -1.231 & .189 & .09 \\
Decisión & 2.43 & .62 & 2.38 & .64 & -1.032 & .294 & .08 \\
Atención & 2.41 & .57 & 2.44 & .59 & -.867 & .392 & .05 \\
Actividad & 2.67 & .57 & 2.54 & .61 & -2.453 & .003 & .22 \\
Afecto & 15.63 & 3.55 & 16.32 & 4.01 & -1.923 & .020 & .18 \\
Negativo & 1.51 & .59 & 1.66 & .64 & -3.100 & .008 & .25 \\
Tensión & 1.52 & .60 & 1.57 & .63 & -1.031 & .276 & .08 \\
Disgusto & 1.57 & .58 & 1.52 & .60 & -1.009 & .199 & .08 \\
Culpa & 1.33 & .56 & 1.44 & .63 & -2.543 & .025 & .18 \\
Susto & 1.62 & .63 & 1.56 & .62 & -1.231 & .188 & .09 \\
Enojo & 1.52 & .62 & 1.57 & .66 & -.765 & .654 & .09 \\
Irritación & 1.60 & .65 & 1.75 & .72 & -1.987. & .013 & .22 \\
Vergüenza & 1.81 & .69 & 1.89 & .73 & -1.237 & .231 & .11 \\
Nerviosismo & 1.73 & .73 & 1.86 & .69 & -2.599 & .012 & .18 \\
Intranquilidad & 1.41 & .62 & 1.49 & .66 & -1.999 & .089 & .12 \\
Temor & & & & & &
\end{tabular}

mediante análisis de varianza (ANOVA) (ver Tabla 2): submuestra de chicos $(\mathrm{N}=406)$ y submuestra de chicas $(\mathrm{N}=380)$.

En este sentido, con respecto a la dimensión $A P$ las puntuaciones medias más elevadas en los chicos en relación a las chicas son: a) energía; b) disposición; c) inspiración; d) decisión; y e) actividad. Mientras que las puntuaciones medias más elevadas en las chicas en relación a los chicos son: a) interés; b) animación; c) entusiasmo; y d) atención. Por otro lado, con respecto a la dimensión $A N$ las puntuaciones medias más elevadas en los chicos en relación a las chicas son: a) culpa; y b) enojo. Mientras que las puntuaciones medias más elevadas en las chicas en relación a los chicos son: a) tensión; b) disgusto; c) susto; d) irritación; e) vergüenza; f) nerviosismo; g) intranquilidad; y h) temor.

En cuanto a las variables $A P$ se hallaron diferencias significativas en función del sexo en las variables, energía y actividad, siendo las puntuaciones de los chicos más elevadas. Para poder valorar la magnitud de estas diferencias se calculó el tamaño del efecto (Cohen, 1994) observándose un tamaño del efecto pequeño, tanto para energía $(d=.18)$ como para actividad $(d=.22)$.

En cuanto a la variable $A N$ se hallaron diferencias significativas en función del sexo en las variables: a) puntuación global de $A N ;$ b) tensión; c) susto; d) vergüenza; y por último e) intranquilidad. Siendo más elevadas las puntuaciones de las chicas. Se observa un tamaño del efecto pequeño en todas las variables del $A N$ : puntación global de $A N(d=.18)$, tensión $(d=.25)$, susto $(d=.18)$, vergüenza $(d=.22)$ e intranquilidad $(d=.18)$. Para una representación gráfica de los resultados véase Figura 1.

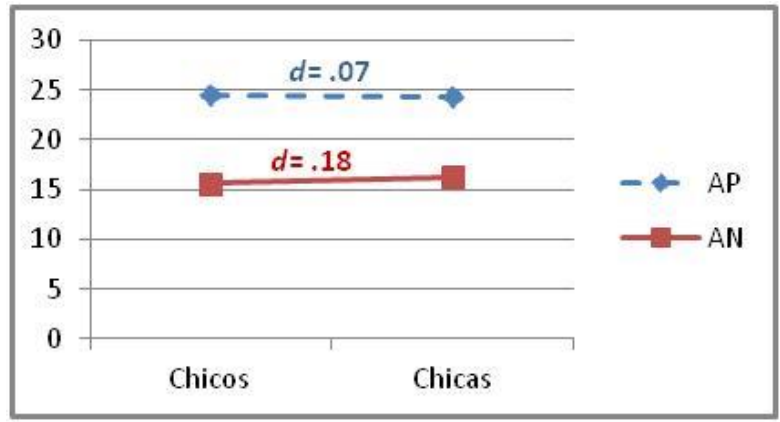

Figura 1. Representación gráfica de las diferencias en afecto positivo y negativo en función del sexo

Para explorar las posibles diferencias de medias en función del IMC del alumnado, se crearon dos subgrupos para su comparación mediante análisis de varianza (ANOVA) (ver Tabla 3): submuestra de alumnos sin problemas de obesidad/sobrepeso $(\mathrm{N}=233)$ y submuestra de 
Tabla 3. Media, desviación típica (DT), ANOVA, y tamaño del efecto de las diferencias de medias (d) en función del IMC de todos los alumnos

\begin{tabular}{lccccccc}
\hline \multicolumn{2}{c}{ Sin obesidad } & \multicolumn{2}{c}{ Con obesidad } & & & \\
\hline Variables & Media & DT & Media & DT & $F$ & sig & $d$ \\
\hline Afecto Positivo & 24.58 & 2.95 & 23.77 & 3.07 & -1.543 & .031 & .27 \\
Interés & 2.41 & .58 & 2.39 & .53 & -.701 & .672 & .04 \\
Animación & 2.46 & .61 & 2.36 & .65 & -1.667 & .172 & .16 \\
Energía & 2.73 & .48 & 2.49 & .59 & -2.998 & .003 & .45 \\
Entusiasmo & 2.27 & .61 & 2.26 & .61 & -.558 & .643 & .02 \\
Orgullo & 2.54 & .56 & 2.42 & .62 & -1.543 & .099 & .20 \\
Disposición & 2.39 & .63 & 2.27 & .68 & -1.453 & .178 & .18 \\
Inspiración & 2.22 & .65 & 2.19 & .62 & -.542 & .887 & .04 \\
Decisión & 2.45 & .61 & 2.43 & .57 & -.332 & .771 & .03 \\
Atención & 2.44 & .57 & 2.36 & .57 & -1.321 & .332 & .14 \\
Actividad & 2.67 & .56 & 2.55 & .62 & -1.678 & .048 & .20 \\
Afecto Negativo & 15.67 & 3.89 & 16.14 & 4.01 & -1.021 & .387 & .12 \\
Tensión & 1.57 & .62 & 1.58 & .65 & -.051 & .974 & .02 \\
Disgusto & 1.49 & .57 & 1.65 & .68 & -1.889 & .059 & .26 \\
Culpa & 1.51 & .60 & 1.58 & .58 & -1.439 & .352 & .12 \\
Susto & 1.36 & .58 & 1.38 & .64 & -.037 & .997 & .03 \\
Enojo & 1.48 & .59 & 1.60 & .61 & -1.876 & .087 & .20 \\
Irritación & 1.56 & .63 & 1.58 & .65 & -.345 & .892 & .03 \\
Vergüienza & 1.68 & .68 & 1.70 & .73 & -.201 & .924 & .03 \\
Nerviosismo & 1.85 & .72 & 1.87 & .73 & -.153 & .887 & .03 \\
Intranquilidad & 1.75 & .70 & 1.77 & .71 & -.298 & .799 & .03 \\
Temor & 1.41 & .61 & 1.43 & .63 & -.301 & .852 & .03 \\
\hline
\end{tabular}

Nota. d=tamaño del efecto de Cohen

alumnos con problemas de obesidad/sobrepeso $(\mathrm{N}=106)$.

En este sentido, con respecto a la dimensión $A P$ todas las puntuaciones medias más elevadas se encuentran en el alumnado sin problemas de obesidad/sobrepeso. Por otro lado, con respecto a la dimensión $A N$ todas las puntuaciones medias más elevadas se encuentran en el alumnado con problemas de obesidad/sobrepeso.

En cuanto a las variables $A P$ se hallaron diferencias significativas en función del IMC en las variables, puntuación global de AP, energía, y actividad a favor de los alumnos sin problemas de obesidad/sobrepeso. Para poder valorar la magnitud de estas diferencias se calculó el tamaño del efecto observándose un tamaño del efecto pequeño, tanto para la puntuación global de AP $(d=.27)$ como para actividad ( $d=.20)$ y un tamaño del efecto moderado para energía $(d=.45)$. En cuanto a la variable AN no se hallaron diferencias significativas en función del IMC en ninguna de las variables del estudio. Para una representación gráfica de los resultados véase Figura 2.

Para explorar las posibles diferencias de medias en función del IMC del subgrupo de los chicos, se crearon dos subgrupos para su comparación mediante análisis de varianza (ANOVA): submuestra de chicos sin problemas de obesidad/

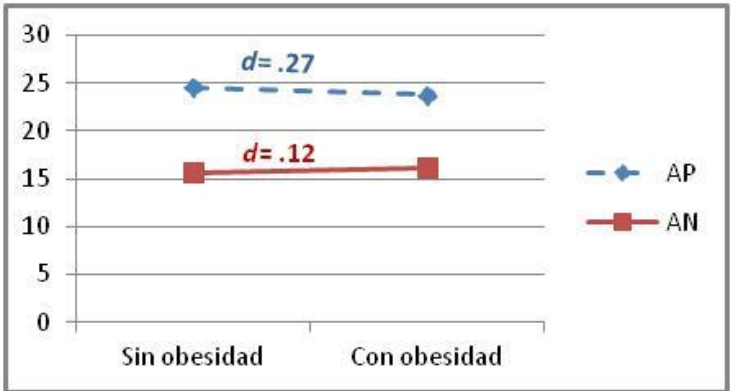

Figura 2. Representación gráfica de las diferencias en afecto positivo y negativo en función del IMC en chicos

sobrepeso $(\mathrm{N}=118)$ y submuestra de chicos con problemas de obesidad/sobrepeso $(\mathrm{N}=73)$. Con respecto a la dimensión $A P$ todas las puntuaciones medias más elevadas se encuentran en los chicos sin problemas de obesidad/sobrepeso exceptuando entusiasmo e inspiración. Por otro lado, con respecto a la dimensión $A N$ todas las puntuaciones medias más elevadas se encuentran en los chicos con problemas de obesidad/sobrepeso.

En cuanto a las variables $A P$ se hallaron diferencias significativas en función del IMC en las variables, energía $(F=-3.283 ; p=.001)$, disposición ( $F=-2.963 ; p=.003)$, y actividad ( $F=$ -2.913; $p=.004)$. En este sentido, las puntuaciones de los chicos sin problemas de obesidad/sobrepeso son mayores que las puntuaciones de los chicos 
con problemas de obesidad/sobrepeso. Para poder valorar la magnitud de estas diferencias se calculó el tamaño del efecto observándose un tamaño del efecto moderado: energía $(d=.46)$, disposición $(d=.47)$ y actividad $(d=.42)$.

En cuanto a la variable AN se hallaron diferencias significativas en función del IMC en los chicos en las variables: a) puntuación global de $A N(F=-2.058 ; p=.041) ; \mathrm{y} \mathrm{b})$ culpa $(F=-2.254$; $p=.024)$. Siendo más elevadas las puntuaciones de los chicos con problemas de obesidad/sobrepeso. Se observa un tamaño del efecto moderado en todas las variables del $A N$ : puntación global de $A N(d=.33)$ y culpa $(d=.34)$.

\section{Diferencias de medias en función del IMC del subgrupo "chicas"}

Para explorar las posibles diferencias de medias en función del IMC del subgrupo de las chicas, se crearon dos subgrupos para su comparación mediante análisis de varianza (ANOVA): submuestra de chicas sin problemas de obesidad/sobrepeso $(\mathrm{N}=115)$ y submuestra de chicas con problemas de obesidad/sobrepeso $(\mathrm{N}=33)$.

En este sentido, con respecto a la dimensión $A P$ todas las puntuaciones medias más elevadas se encuentran en las chicas sin problemas de obesidad/sobrepeso exceptuando entusiasmo y disposición. Igualmente, con respecto a la dimensión $A N$ todas las puntuaciones medias más elevadas se encuentran en las chicas sin problemas de obesidad/sobrepeso exceptuando disgusto e irritación.

En cuanto a las variables $A P$ se hallaron diferencias significativas en función del IMC de las chicas en las variables, animación ( $F=-2.157$; $p=.031)$, y energía $(F=-3.078 ; p=.002)$. En este sentido, las puntuaciones de las chicas sin problemas de obesidad/sobrepeso son mayores que las puntuaciones de las chicas con problemas de obesidad/sobrepeso. Para poder valorar la magnitud de estas diferencias se calculó el tamaño del efecto observándose un tamaño del efecto moderado: animación $(d=.45)$ y energía $(d=.65)$.

En cuanto a la variable $A N$ no se hallaron diferencias significativas en función del IMC en las chicas en ninguna de las variables del estudio (Figura 3).

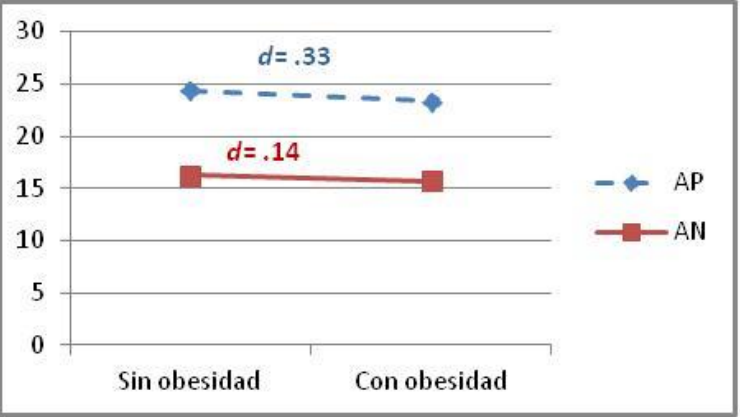

Figura 3. Representación gráfica de las diferencias en afecto positivo y negativo en función del IMC en chicas

\section{Discusión}

En primer lugar, los resultados confirmaron que las puntuaciones son más elevadas en $A P$ que en $A N$. Este patrón se ha observado previamente en anteriores hallazgos con muestras de adultos (Sandín et al, 1999) y con muestras de niños y adolescentes (Barrón-Sánchez \& Molero, 2014; Sandín, 2003). Aunque los resultados evidencian una puntuación ligeramente más elevada en $A P$ y una puntuación más baja en $A N$ que las obtenidas por Sandín (2003). En este sentido, probablemente la clase de Educación Física puede ofrecer al alumnado, en el contexto escolar, la oportunidad de manifestar emociones muy variadas con un patrón que se caracteriza por la disminución del $A N$ y el aumento del AP (Durán et al., 2015; Ferrer-Caja \& Weiss, 2000; Moreno-Murcia \& Hernández, 2009). Por otro lado, las correlaciones entre las dimensiones de $A P$ y AN fueron muy bajas y en la dirección esperada $(r=-.08 ; p=.05)$ corroborando que el AP y el AN tienden a correlacionar ligeramente entre sí de forma negativa, confirmando así la bidimensionalidad del PANAN.

En segundo lugar, los resultados evidencian diferencias significativas entre chicos y chicas en $A P$ en las variables energía y actividad. En este sentido, los chicos presentan más $A P$ que las chicas en la clase de Educación Física especialmente en las variables que más contribuyen al factor de $A P$ y que se constituyen como verdaderos indicadores del AP: energía y actividad (Sandín, 1999, 2003). Igualmente, los resultados confirman diferencias significativas entre chicos y chicas en $A N$ en las variables: puntuación global de $A N$, tensión, susto, 
vergüenza e intranquilidad. Así pues, las chicas presentan más $A N$ que los chicos en la clase de educación física. Nuestros resultados replican la asociación entre medidas de autoinforme de emocionalidad negativa y sexo femenino (Etxeberría et al., 2008; Gil-Madrona, CachónZagalaz, Díaz-Suarez, Valdivia-Moral, \& Zagalaz-Sánchez, 2014). Por tanto, estos resultados pueden aportar información a los docentes para el diseño y desarrollo de programas de intervención en la clase de educación física que sean adecuados a las características emocionales de las alumnas con la finalidad de disminuir las emociones negativas y aumentar las positivas teniendo en cuenta que el disfrute es una condición indispensable para practicar deporte en el futuro (Ntoumanis et al., 2004; González-Cutre et al., 2008; Yli-Piipari et al., 2009), y además tal y como se ha resaltado anteriormente los afectos positivos en la actividad física pueden consolidar conductas asociadas a un estilo de vida saludable (Pieron et al., 2007).

En tercer lugar, en relación con el análisis sobre las diferencias en función del IMC del alumnado es reseñable que en la dimensión $A P$ todas las puntuaciones medias más elevadas se encuentran en el alumnado sin problemas de obesidad/sobrepeso. Asimismo, con respecto a la dimensión $A N$ todas las puntuaciones medias más elevadas se encuentran en el alumnado con problemas de obesidad/sobrepeso. En este sentido, los resultados evidencian diferencias significativas en función del IMC entre alumnos con problemas de sobrepeso/obesidad y alumnos sin problemas de sobrepeso/obesidad en $A P$ en las variables: puntuación global de AP, energía y actividad. Por otro lado, no existen diferencias significativas en $A N$. Una posible explicación de estos resultados podría estar relacionada con el impacto que la obesidad presenta sobre algunos estados emocionales negativos tales como, altos niveles de ansiedad, depresión, insatisfacción con la imagen corporal y bajo autoconcepto (Ross, 1994; Friedman \& Brownell, 1995). De hecho, MorenoEsteban y Andía-Melero (2009), señalan que la relación entre el exceso de peso corporal y el malestar psicoemocional condiciona el nivel de satisfacción con la vida, ya que la presión social para ser delgado afecta cada vez más y de manera significativa a las personas obesas.
Por último, es destacable que tanto los chicos como las chicas sin problemas de obesidad/sobrepeso presentan más emociones positivas que los chicos y chicas con problemas de obesidad/sobrepeso, lo que se encuentra en la línea de los resultados globales anteriores. No obstante, en cuanto a las emociones negativas, se confirma un patrón diferenciado entre chicos y chicas. Los chicos con problemas de obesidad/sobrepeso tienen puntuaciones significativamente más elevadas en AN que los chicos sin problemas de obesidad/sobrepeso, y por tanto, el grado de malestar es mayor en las clases de Educación Física mientras que este patrón de respuesta no se presenta en las chicas. Estos resultados probablemente puedan ser explicados por la influencia de ciertos estereotipos de género en la clase de Educación Física. En este sentido, los chicos, en comparación con las chicas, otorgan más importancia a determinadas actividades competitivas realizadas en la clase de Educación Física y además perciben que el éxito en estas actividades influye en su estatus social y educativo (Vera, Moreno, \& Moreno, 2009). Desde estos planteamientos, es factible pensar que la clase de Educación Física puede ser un contexto adecuado para fomentar actitudes no sexistas y que propicien la coeducación.

A pesar de estos resultados que pudieran ser útiles para la enseñanza de la Educación Física, reconocemos varias limitaciones en el presente estudio. La primera hace referencia al carácter no aleatorio de la muestra empleada, que limita su representatividad $\mathrm{y}$, por tanto, debemos ser precavidos a la hora de generalizar estos resultados. La segunda se refiere a que la muestra pertenece exclusivamente a una comunidad autónoma y no sabemos si en otro marco sociocultural modificaría los resultados. Para subsanar estas limitaciones el equipo investigador tiene en perspectiva llevar a cabo una ampliación de este estudio contando con una muestra en la que participen orientadores en ejercicio de varias comunidades autónomas. Como líneas futuras de investigación se puede sugerir la exploración de otras variables, como la motivación o el rendimiento académico obtenido.

En conclusión, estos resultados pueden aportar información a los docentes para el diseño y desarrollo de programas de intervención para 
atender a la diversidad de características del alumnado especialmente para que en la programaciones didácticas y las diferentes actuaciones educativas de los centros escolares contengan el disfrute como una condición indispensable para practicar deporte (GonzálezCutre et al., 2008; Ntoumanis et al., 2004; YliPiipari et al., 2009) y además, tal y como se ha resaltado anteriormente, se fomente el desarrollo de afectos positivos que menos destacan en los alumnos y disminuir los aspectos negativos que más registran los chicos y las chicas y que se han asociado al IMC inscritos a la actividad física para consolidar conductas relacionadas con un estilo de vida saludable (Pieron et al., 2007: Spence y Blanchard, 2001). En definitiva, este estudio contribuirá realmente a la finalidad principal de la Educación Física en su contribución al desarrollo integral de la personalidad del alumnado y para llevar a cabo una educación física emocional, tal y como proponen Miralles, Filella y Lavega (2017).

\section{Referencias}

Bailey, R. (2006). Physical Education and sports in schools: A review of benefits and outcomes. Journal of School Health, 76(8), 397-401. doi:10.1111/j.1746-1561.2006.00132.x

Barrón-Sánchez, M. C., \& Molero, D. (2014). Estudio sobre inteligencia emocional y afectos en escolares de educación primaria. Revista Electrónica de Investigación y Docencia, 8, 718.

Bar-On, R. (2006). The Bar-On model of emotional-social intelligence (ESI). Psicothema, 18 (supl), 13-25.

Bar-On, R. (2010). Emotional intelligence: An integral part of positive psychology. South African Journal of Psychology, 40(1), 54-62. doi:10.1177/008124631004000106

Bausela-Herreras, E. (2018). PISA 2012: Ansiedad y Bajo Rendimiento en Competencia Matemática. Revista Iberoamericana de Diagnóstico y Evaluación - e Avaliação Psicológica, 46(1), 161-173. doi:10.21865/RIDEP46.1.12

Bhullar, N., Schutte, N. S., \& Malouff, J. M. (2012). Associations of individualistic collectivistic orientations with emotional intelligence, mental health, and satisfaction with life: A tale of two countries. Individual Differences Research, 10(3), 165-175.

Biro, F. M., \& Wien, M. (2010). Childhood obesity and adult morbidities. The American Journal of Clinical Nutrition, 91(5), 1499S1505S. doi:10.3945/ajen.2010.28701B

Bisquerra, R. (2003). Educación Emocional. Programa para educación primaria (6-12 años). Barcelona. Wolters Kluwer.

Bisquerra, R. (2011). Educación Física, competencias básicas y educación emocional. EmásF, Revista digital de Educación Física, 11, 4-6.

Bisquerra, R., Pérez-González, J. C., \& García, E. (2015). Inteligencia emocional en educación. Madrid: Síntesis.

Brackett, M. A., \& Mayer, J. D. (2003). Convergent, discriminant, and incremental validity of competing measures of emotional intelligence. Personality and Social Psychology Bulletin, 29 (10), 1147-1158. doi:10.1177/0146167203254596

Brasseur, S., \& Georgoire, J. (2011). L'inteligence émotionelle-Trait chez les adolescents á haut potential: Spédifities et liens avec réussite scolaire et les competences socials. Enfance, 62(1) 59-76.

Cárdenas-Cárdenas, L. M., Burguete-García, A. I., Estrada-Velasco, B. I., López-Islas, C., Peralta-Romero, J., Cruz, M. \& GalvánPortillo, M. (2015). Leisure-time physical activity and cardiometabolic risk among children and adolescents. Journal de Pediatría, $\quad$ 91(2), 136-142. doi:10.1016/j.jped.2014.06.005

Chan, D. W. (2006). Emotional intelligence and components of burnout among Chinese secondary school teachers in Hong Kong. Teaching and Teacher Education, 22, 10421054. doi:10.1016/j.tate.2006.04.005

Cohen, J. (1994). The earth is round $(p<.05)$. American Psychologist, 49 (12), 997-1003.

Cox, A. E., Smith, A. L., \& Williams, L. (2008). Change in physical education motivation and physical activity behavior during middle school. Journal of Adolescent Health, 43, 506513. doi:10.1016/j.jadohealth.2008.04.020

Cox, A. E., \& Ullrich-French, S. (2010). The motivational relevance of peer and teacher 
relationship profiles inphysical education. Psychology of Sportand Exercise, 11, 337344. doi:10.1016/j.psychsport.2010.04.001

Donnelly, J. E., Blair, S. N., Jakicic, J. M., Manor, M. M., Rankin, J. W., \& Smith, B. K. (2009). Appropriate physical activity intervention strategies for weight loss and prevention of weight regain for adults. Medicine and Science in Sports and Exercise, 41(2), 459471. doi:10.1249/MSS.0b013e3181949333

Durán, C., Lavega, P., Salas, C., Tamarit, M. \& Invernó, J. (2015). Educación física emocional en adolescentes. Identificación de variables predictivas de la vivencia emocional. Cultura, Ciencia y Deporte (CCD), 10(28), 5-18.

Durlak, J. A., Weissberg, R. P., Dymnicki, A. B., Taylor, R. D., \& Schellinger, K. B. (2011). The impact of enhancing students' social and emotional learning: A meta-analysis of school-based universal interventions. Child Development, $\quad 82, \quad$ 405-432. doi:10.1111/j.1467-8624.2010.01564.x

Espada, M. \& Calero, J. C. (2012). La inteligencia emocional en el área de Educación Física. La peonza. Revista de Educación Física para la paz, 7, 65-69.

Etxebarría, I. Aritzeta, A., Barberá, E., Chóliz, M., Jiménez, M. P., Martínez-Sánchez, F., Mateos, P., \& Páez, D. (Eds). (2008). Emoción y Motivación. Contribuciones actuales. Vol. 2: Motivación. San Sebastián: AME.

Friedman, M. A., \& Brownell, K. D. (1995). Psychological correlates of obesity: Moving to the next research generation. Psychological Bulletin, 117(1), 3-20.

García, A., \& Bores, N. (2004). La expresión corporal en la educación física obligatoria. [en línea] [Disponible en http://www.expresiva.org/AFYEC/Articulos /X016_La_EC_en_EF_obligatoria.pdf]

[Consulta: 4-3-2016)

Gil-Madrona, P., \& Contreras, O. (2003). Interés y valoración del área de Educación Física por padres y alumnos en la enseñanza obligatoria. Revista de Educación, 332, 255-283

Gil-Madrona, P., Cachón-Zagalaz, J., DíazSuarez, A., Valdivia-Moral, P., \& ZagalazSánchez, M. L. (2014). Las niñas también quieren jugar: La participación conjunta de niños y niñas en actividades físicas no organizadas en el contexto escolar. Movimento 20(1), 103-124. doi:10.22456/1982-8918.38070

Gil-Madrona, P.,,, \& Martínez-López, M. (2016). Emociones percibidas, por alumnos y maestros, en Educación Física en 6. ${ }^{\circ}$ curso de primaria. Educación XX1, 19(2), 179-204. doi:10.5944/educxx1.16460

González, C., \& Lleixa, A. (2010). Didáctica de la Educación Física. 4. Vol. II. Barcelona. Grao.

González-Cutre, D., Sicilia, A., \& Moreno, J.A. (2008). Modelo cognitivo-social de la motivación de logro en educación física. Psicothema, 20(4), 642-651

Gutiérrez, M. (2000). Actividad física, estilos de vida y calidad de vida. Revista de Educación, 77, 5-14.

Hashim, H., Grove J. R., \& Whipp, P. (2008). Validating the youth sport enjoyment construct in high school physical education. Research Quarterly for Exercise and Sport, 79, 183-195.

Hausenblas, H. A., \& Fallon, E. A. (2006). Exercise and body image: A meta-analysis. Psychology and Health, 21, 33-47. doi:10.1080/14768320500105270

Lagardera, F., \& Lavega, P. (2003). Introducción a la praxiología motriz. Barcelona: Paidotribo.

Lavega, P., Filella, G., Lagardera, F., Mateu, M., \& Ochoa, J. (2013). Juegos motores y emociones. Cultura y Educación (C\&E), 25(3), 347-360. doi:10.1174/113564013807749731

Lavega, P., March, J., \& Filella G. (2013). Juegos deportivos y emociones. Propiedades psicométricas de la escala GES para ser aplicada en la Educación Física y el Deporte. Revista de Investigación Educativa, RIE 31(1), 151-166. doi:10.6018/rie.31.1.147821

Manríquez-Betanzos, J. A., \& Montero-López, A. (2018). Validación de la Escala de Emociones hacia el Cuidado del Agua, Revista Iberoamericana de Diagnóstico y Evaluación - e Avaliação Psicológica. 46(1), 147-159. doi:10.21865/RIDEP46.1.11

Malik, V. S., Willett, W. C., \& Hu, F. B. (2013). Global obesity: Trends, risk factors and policy 
implications. Nature Reviews Endocrinology, 9, 13-27. doi:10.1038/nrendo.2012.199

Marchesi, A., \& Hernández, C. (2003). El fracaso escolar. Perspectiva internacional. Madrid: Alianza.

McCaughtry, N. (2004). The emotional dimensions of a teacher`s pedagogical content knowledge: Influences on content, curriculum, and pedagogy. Journal of Teaching in Physical Education, 23(1), 30-47. doi:10.1123/jtpe.23.1.30

Miralles, R.; Filella, G., \& Lavega, P. (2017). Educación física emocional a través del juego en educación primaria. Ayudando a los maestros a tomar decisiones. Retos, 31, 88-93

Morano, M., Colella, D., \& Capranica, L. (2011). Body image, perceived and actual physical abilities in normal-weight and overweight boys involved in individual and team sports. Journal of Sports Sciences, 29(4), 355-362. doi:10.1080/02640414.2010.530678

Moreno-Esteban, B. \& Andía-Melero, V.M. (2009). Tratamiento de la obesidad. Revista Siete Dias Médicos, 778, 34-38.

Motos, T. (2003). Cerebro emocional, educación emocional y expresión corporal. Actas I Congreso Internacional de Expresión Corporal y Educación. Universidad Europea de Madrid. Salamanca: Amarú Ediciones.

Mujica, F. N., Orellana, N. C., \& Concha, R. F. (2017). Emociones en la clase de Educación Física: Revisión narrativa (2010-2016). Ágora para la Educación Física y el Deporte 19(1), 119-134. doi:10.24197/aefd.1.2017.119-134

Ogden, C. L., Carroll, M. D., Kit, B. K., \& Flegal, K. M. (2012). Prevalence of obesity and trends in body mass index among US children and adolescents, 1999-2010. The Journal of the American Medical Association, 307(5), 483-490. doi:10.1001/jama.2012.40

Parlebas, P. (2001). Juegos, deporte y sociedad. Léxico de praxiología motriz. Barcelona: Paidotribo

Pellicer, I. (2011). Educación Física Emocional. De la teoría a la práctica. Barcelona: Inde.

Pena, M., Extremera, N., \& Rey, L (2011). El papel de la inteligencia emocional en la resolución de problemas sociales en estudiantes adolescentes. Revista Española de Orientación y Psicopedadogía, 22, 69-79.
Perez-Farinos, N., López-Sobaler, A., Ángeles Dal Re, M., Villar, C., Labrado, E., Robledo, T., \& Ortega, R. (2013). The ALADINO Study: A national study of prevalence of overweight and obesity in Spanish children in 2011. BioMed Research International. Volume 2013, Article ID 163687, 7 pages. doi:10.1155/2013/163687

Perry, A. (2013). Presentación, en P. Gil-Madrona (coord) Desarrollo curricular de la Educación Física en la Educación Infantil. Madrid: Pirámide.

Pieron, M., García Montes, M. E., \& Ruiz, F. (2007). Algunos correlatos de la actividad físico-deportiva en una perspectiva de salud. Tándem. Didáctica de la Educación Física, 24, 9-24.

Roberts, G. (1992). Motivation in sport and exercise: Conceptual constraints and convergence. In G. Roberst (Ed), Motivation in sport and exercise (pp. 3-29). Champaign, IL: Human Kinetics.

Ross, C. E. (1994). Overweight and depression. Journal of Health and Social Behavior, 35(1), 63-79.

Ruiz, G., Lorenzo, L., \& García, A. (2013). El trabajo con la inteligencia emocional en las clases de Educación Física: Valoración de una experiencia piloto en Educación Primaria. Journal of Sport and Health Research. 5(2), 203-210.

Sánchez, J. J., Jiménez, J. J., Fernández, F., \& Sánchez, M. J. (2013). Prevalencia de obesidad infantil y juvenil en España en 2012. Revista Española de Cardiología, 66(5), 371376. doi:10.1016/j.recesp.2012.10.016

Sandín, B. (2003). Escalas PANAS de afecto positivo y negativo para niños y adolescentes (PANASN). Revista de Psicopatología y Psicología Clínica, 8, 173-182.

Sandín, B., Chorot, R, Lostao, L., Joiner, T. E., Santed, M. A., \& Valiente, R. M. (1999). Escalas PANAS de afecto positivo y negativo: Validación factorial y convergencia transcultural. Psicothema, 2, 37-51.

Scrabis-Fletcher, K., \& Silverman, S. (2010). Perception of competence in Middle School physical education: Instrument development and validation. Research Quarterly for 
Exercise and Sport, 81(1), 53-61. doi:10.1080/02701367.2010.10599627

Strong, W. B., Malina, R. M., Blimkie, C. J., Daniels, S. R., Dishman, R. K., Gutin, B. et al. (2005). Evidence based physical activity for school-age youth. The Journal of Pediatrics, 146, 732-737. doi:10.1016/j.jpeds.2005.01.055

Van Acker, R., Carreiro da Costa, F., De Bourdeaudhuij, I., Cardon, G., \& Haerens, L. (2010). Sex equity and physical activity levels in coeducational Physical Education: exploring the potential of modified game forms. Physical Education and Sport Pedagogy, 15, 159-173.

Vera, J.A., Moreno, R., \& Moreno, J.A. (2009). Relación entre la cesión de responsabilidad y la percepción de igualdad en la enseñanza de la educación física escolar. Cultura, Ciencia y Deporte, 6, 25-31.

Watson, D., Clark, L. A., \& Tellegen, A. (1988). Development and validation of brief measures of positive and negative affect: The PANAS scales. Journal of Personality and Social Psychology, 54, 1063-1070.

WHO (World Health Organization) (2010). Global Recommendations on Physical Activity for Health. Geneva: World Health Organization; 2010.

Yli-Piipari, S., Watt, A., Jaakkola, T., Liukkonen, J., \& Nurmi, J-E. (2009). Relationships between physical education students' motivational profiles, enjoyment, state anxiety, and selfreported physical activity. Journal of Sports Science and Journal and Medicine 8, 327-336.

Zach, S., Zeev, A., Dunsky, A., Goldbourt, U., Shimony, T., Goldsmith R., \& Netz, R. (2013). Perceived body size versus healthy body size and physical activity among adolescents - Results of a national survey. European Journal of Sport Science. [en línea]. Disponible en http://sport-science.orgdoi:10.1080/17461391.2013.77138

Zamorano-García, M., Gil-Madrona, P., PrietoAyuso, A., \& Zamorano-García, D. (2018). Emociones generadas por distintos tipos de juegos en clase de educación física. Revista Internacional de Medicina y Ciencias de la Actividad Física y el Deporte. 18(69), 1-26. doi:10.15366/rimcafd2018.69.001 